\title{
The Top Articles in Geriatrics 2016-2017*
}

\author{
Kenneth Lam, MD, FRCPC, Dov Gandell, MDCM, FRCPC \\ Department of Medicine, Division of Geriatrics, University of Toronto, Toronto, ON, Canada
}

DOI:https://doi.org/10.5770/cgj.21.330

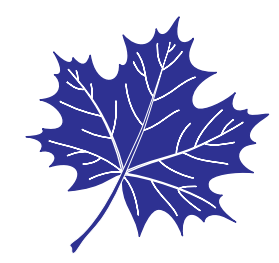

\begin{abstract}
We present the top 11 articles in geriatric medicine in the past two years. The topics range from new diagnostic criteria for Lewy Body dementia, advances in biomarker diagnosis of Alzheimer's disease, a major review of dementia and risk factors, the optimal cutoff for the Montreal Cognitive Assessment, antipsychotics in delirium prevention, mobilization of inpatients, intensive blood pressure treatment and statin therapy for primary prevention in older adults, and comprehensive geriatric assessment in vascular surgery and non-small cell lung cancer.
\end{abstract}

Key words: geriatrics, literature update, 2017, dementia, delirium, hypertension, hyperlipidemia, inpatient mobilization, comprehensive geriatric assessment

\section{Introduction}

We present the top 11 articles in geriatric medicine in the past two years. In the summer of 2017, the second author (DG) surveyed geriatricians $(\mathrm{N}=51)$ at the University of Toronto by e-mail requesting articles they considered important to the field of geriatrics, published in the last year without any further specific criteria. Five responses with suggestions for 33 articles were obtained. The second author also hand-searched major general medicine and geriatric journals, including the New England Journal of Medicine, the Journal of the American Medical Association, Neurology, the Journal of the American Geriatric Society, Age and Ageing, the Canadian Journal of Geriatrics, and the Journals of Gerontology. The final selection of articles chosen was achieved by consensus between the two authors with the criterion being the possibility of influencing practice or the understanding of practice. We include four articles on cognitive impairment and dementia, one article on delirium, one article on in-patient mobilization, two articles on blood pressure management, one article on lipid management, and two articles on the use of the comprehensive geriatric assessment.

\section{REVIEW OF THE TOP 11 ARTICLES}

\section{The diagnostic criteria for Lewy Body dementia have been revised, with refined clinical criteria and the inclusion of diagnostic biomarkers.}

McKeith IG, Boeve BF, Dickson DW, et al. Diagnosis and management of dementia with Lewy bodies. Neurology. 2017;89(1):88-100.

\section{Background}

The Dementia with Lewy Bodies (DLB) Consortium last met in 2005 and established a framework for defining Lewy Body dementia as a dementia syndrome with core features (fluctuating cognition, recurrent visual hallucinations, and parkinsonism) and suggestive features (REM sleep behaviour disorder, severe neuroleptic sensitivity, and low dopamine transporter on imaging). ${ }^{(1)}$ However, detection rates in clinical practice remained low. Lewy body disease biomarker development now enables refinement of the clinical diagnosis of DLB by incorporating clinical features with diagnostic biomarkers which may increase clinical sensitivity.

\section{Methods}

Working groups on clinical diagnosis, management, basic science, and global harmonization were established. A conference of international experts was held in December 2015 to reach consensus.

\section{Findings}

The 2017 revision maintains the essential criterion: that of progressive cognitive decline of sufficient magnitude to interfere with normal social function, occupational function, or usual daily activities. The revised criteria now diagnose probable DLB based on the presence of two or more core clinical features, or one core clinical feature with one indicative biomarker. The core clinical features include a new criterion, REM sleep behaviour disorder. Inquiry into prior sleep disturbances (dreams with a chasing or attacking theme, limb movements noted by a bed partner) should be included in all patients with new onset dementia. The previous three core criteria-1) fluctuating cognition with pronounced variations in attention and alertness; 2) recurrent visual hallucinations

\footnotetext{
*The content was previously presented at the 2017 Toronto Geriatrics
} Update Course on 27 October 2017. 
that are typically well-formed and detailed; and 3) one or more spontaneous cardinal features of parkinsonism - remain the same. Indicative biomarkers have replaced suggestive features in the criterion-based diagnosis. They include low dopamine transporter uptake in basal ganglia on SPECT or PET (sensitivity $78 \%$, specificity $90 \%$ ), low uptake of iodine-MIBG myocardial scintigraphy (sensitivity $69 \%$, specificity $87 \%$ ), and polysomnographic confirmation of REM sleep without atonia (PPV $>90 \%$ with dementia and RBD). Suggestive features (e.g., neuroleptic hypersensitivity, hyposmia, relative preservation of medial temporal lobe structures on $\mathrm{CT} / \mathrm{MRI}$ ) have been re-categorized as supportive clinical features or supportive biomarkers and are not formally included in the diagnostic criteria due to a lack of specificity.

\section{Cautions}

Aside from polysomnogram investigation, the other new biomarkers are not widely available. Pharmacologic management of DLB is still limited to cholinesterase inhibitors, a treatment that confers modest symptomatic improvement.

\section{Implications}

More sensitive and specific diagnostic criteria will help raise awareness and help identify more cases of DLB.

In the search to identify the underlying pathophysiology of Alzheimer's dementia, the NIA-AA clinical and biomarker-based diagnostic classification may have inappropriately grouped biomarkers of tau and neurodegeneration.

Jack CR Jr., Wiste HJ, Weigand SD, et al. Age-specific and sex-specific prevalence of cerebral $\beta$-amyloidosis, tauopathy, and neurodegeneration in cognitively unimpaired individuals aged 50-95 years: a cross-sectional study. Lancet Neurol. 2017;16(6):435-44.

\section{Background}

The post-mortem histopathologic diagnosis of Alzheimer's disease is based on the presence of amyloid plaques and neurofibrillary tangles composed of hyperphosphorylated tau protein. In 2011, the National Institute of Aging-Alzheimer's Association recommended the use of two biomarkers to aid earlier diagnosis of Alzheimer's disease: 1) amyloid (based on CSF A 342 or PET scan) and 2) tau-related neurodegeneration or neuronal injury (based on CSF phosphorylated tau, MRI or FDG PET). ${ }^{(2)}$ They proposed a system (the AN system) to classify individuals into amyloid abnormal $(\mathrm{A}+)$, amyloid normal (A-), neurodegeneration abnormal $(\mathrm{N}+)$ or neurodegeneration normal (N-) categories to help researchers communicate their findings. A group of patients at risk for developing Alzheimer's disease who were amyloid biomarker negative (A-N+, labelled as suspected non-Alzheimer pathology or SNAP) emerged. To better characterize this A-N+ group there may be a benefit to distinguishing tau pathologies (based on CSF phosphorylated tau) from neurodegeneration (based on MRI or FDG PET).

\section{Methods}

This cross-sectional study randomly selected 435 participants enrolled in the Mayo Clinic Study of Aging, aged 50 to 89, and included those clinically judged to be without cognitive impairment and who had undergone amyloid PET, tau PET, and MRI between October 2006 and October 2016. Participants were classified based on the presence or absence of amyloid $(\mathrm{A}+$ or $\mathrm{A}-)$, tau ( $\mathrm{T}+$ or $\mathrm{T}-)$, and neurodegeneration $(\mathrm{N}+$ or $\mathrm{N}-)$ abnormalities on imaging to produce eight distinct "ATN" states (e.g., A-T-N-, or A-T-N+, etc.)

\section{Findings}

A-T-N- was the most prevalent category until the patient was in his/her late 70 s and decreased with age, whereas $\mathrm{A}+\mathrm{T}+\mathrm{N}+$ prevalence began increasing by the late $60 \mathrm{~s}$ and became the most prevalent from the early 80 s onwards. Tau and neurodegeneration were discordant (e.g., $\mathrm{T}-\mathrm{N}+$ or $\mathrm{T}+\mathrm{N}-$ ) in $86 \%$ of individuals at age 65 and $51 \%$ of individuals at age 80 .

\section{Cautions}

This study did not examine cognitively impaired adults and was a cross-sectional population analysis rather than a longitudinal observation of individual participant trajectories. The authors are clear the ATN classification is a proposed classification framework and not yet supported by evidence to function as a diagnostic or prognostic tool.

\section{Implications}

As many cognitively unimpaired adults in this cohort demonstrated tauopathy on PET imaging without neuronal degeneration and neurodegeneration without tauopathy, the ATN classification system for biomarkers is a useful advance in the understanding of the molecular underpinnings of dementia. Given the heterogeneity of pathological findings on post-mortem examination in older adults, a classification system incorporating biomarkers for vascular and Lewy Body pathology, in addition to ATN, may one day be useful.

A comprehensive review of dementia synthesizes current knowledge on dementia and estimates a third of cases are potentially preventable.

Livingston G, Sommerlad A, Orgeta V, et al. Dementia prevention, intervention, and care. Lancet. 2017; 390(10113):2673-734. Epub 2017 Jul 20. 


\section{Background}

The Lancet Commission on Dementia Prevention, Intervention and Care met to consolidate knowledge on dementia, including its prevalence, risk factors, prevention, treatment, management of psychosocial impact, and potential future directions.

\section{Methods}

They completed a systematic review and meta-analysis to identify risk factors for dementia. They calculated the population attributable fraction based on the prevalence of the risk factor (from the 2014 Health Survey for England) and the strength of the association (based on the metaanalysis), adjusting for communality. The remainder of the article was a comprehensive synthesis of guidelines and other systematic reviews.

\section{Findings}

Potentially modifiable risk factors (with the percentage of dementia cases attributable to that risk factor) were ApoE $\varepsilon 4$ allele (7\%), less education (8\%), hearing loss $(9 \%)$, hypertension $(2 \%)$, obesity $(1 \%)$, smoking $(5 \%)$, depression $(4 \%)$, physical inactivity $(3 \%)$, social isolation $(2 \%)$, and diabetes $(1 \%)$. Other topics covered in the review included prevention and pharmacological treatment, and an emphasis on caring for family members, advanced planning, managing safety and neuropsychiatric symptoms, and end-of-life care.

\section{Cautions}

Few randomized controlled trials are available to demonstrate causality between interventions on risk factors and development of dementia. As such, their estimate of preventable cases of dementia generously assumes that the risk factor is entirely removed and that the relationship with dementia is causal.

\section{Implications}

This article identifies a quantifiable estimate of risk associated with several patient factors that are potentially modifiable. Identifying and improving those risk factors in individuals (for example, hearing loss in mid-life) may have a dramatic effect on reducing their chances of developing dementia.

\section{A meta-analysis of nine studies concludes an optimal cut-off for the MoCA examination should be $<23$ in patients without major comorbidities to reduce false- positive screens.}

Carson N, Leach L, Murphy KJ. A re-examination of Montreal Cognitive Assessment (MoCA) cutoff scores. Int J Geriatr Psychiatry. 2018;33(2):379-88. Epub 2017 Jul 21.

\section{Background}

The original study of the MoCA $(\mathrm{n}=184)$ demonstrated high sensitivity $(90 \%)$ and specificity $(87 \%)$, but real-world usage and later studies suggest older age and lower education produce false-positives with the standard cutoff of $<26$ for detecting mild cognitive impairment (MCI).

\section{Methods}

This meta-analysis found nine studies including 1,048 patients reporting sensitivity and specificity for one or more cut-off points for the diagnosis of MCI. They excluded studies in populations of patients with specific medical, neurological or psychiatric comorbidities, as well as studies without a comparison cohort. The gold standard comparator for all included studies was clinical assessment for MCI as defined by the Petersen criteria (subjective memory complaint or cognitive complaint, impaired memory or other cognitive domain, preserved general cognitive function, functional independence, no dementia). ${ }^{(3)}$ The sensitivity, specificity, positive predictive accuracy, negative predictive accuracy, negative and positive likelihood ratios, and classification accuracy were aggregated and summarized for each cut-off point tested.

\section{Findings}

The MoCA was $100 \%$ sensitive for scores 28 and greater, and maximally specific (98\%) for scores 20 and below. Four studies $(n=370)$ contributed to determining an optimal cutoff point of 23, defined as the highest classification accuracy (based on a Youden index, sensitivity + specificity $-1=0.71$ ) with a sensitivity of $83 \%$ and a specificity of $88 \%$. The positive likelihood ratio for scores below 23 was 7.09 and negative likelihood ratio for scores of 23 and higher was 0.19 .

\section{Cautions}

Most of the studies that contributed to the cut-off score of 23 were not conducted in English. Furthermore, this analysis found few and poor-quality data correlating age or education to the MoCA test score.

\section{Implications}

A cut-off of $<26$ remains very sensitive to detect MCI. In non-English speaking individuals, a cut-off score of $<23$ can be considered abnormal. To improve classification accuracy for the broader population, more normative data across ranges of age and education are still needed.

Haloperidol was not effective in preventing delirium in at-risk hospitalized older patients. 
Schrijver EJM, De Vries OJ, van de Ven PM, et al. Haloperidol versus placebo for delirium prevention in acutely hospitalised older at risk patients: a multi-centre double-blind randomised controlled clinical trial. Age Ageing. 2018;47(1):48-55. Epub 2017 Jul 15.

\section{Background}

Haloperidol has not been shown to prevent delirium in hospitalized older adults.

\section{Methods}

This multi-centre, double-blind, stratified, block-randomized, placebo-controlled trial recruited 245 patients over the age of 70 (mean age 83.5) from six Dutch hospitals admitted through the emergency department, with $75 \%$ admitted to a medical ward and $25 \%$ to a surgical ward. Prior to admission, $45 \%$ were independent at home, $50 \%$ had memory complaints, and $71 \%$ used a gait aid. All screened positive for delirium risk (answered yes to at least one of the following questions: Do you have memory problems? Were you in need for self-care help in the last 24 hours? During previous admissions or illness, did you experience a transient state of confusion?).

(4) Patients were assessed within 24 hours of admission and received haloperidol $1 \mathrm{mg} \mathrm{PO}$ or placebo at 12 p.m. or 8 p.m. for up to one week. Primary outcome was incident delirium based on DSM-IV criteria by experienced clinicians.

\section{Findings}

Incident delirium occurred in $19.5 \%$ of the group treated with haloperidol and $14.5 \%$ in the group who received placebo, with a non-significant difference in odds between groups (OR $1.43,95 \%$ CI $0.72-2.78, p=.302$ ). In the 23 patients in the haloperidol arm and 18 patients in the placebo arm who still developed delirium, similar percentages received open-label haloperidol as treatment $(65.2 \%$ vs. $66.7 \%, p=.923)$. There was no difference between groups in the delirium duration or severity length of stay or three-month mortality. Adverse events (including cardiac complications) were uncommon and comparable in both groups.

\section{Cautions}

The study did not meet the intended sample size, recruiting only $245(63 \%)$ of the intended 390 participants, for a study power of $59 \%$, increasing the possibility of a falsenegative result.

\section{Implications}

Based on the results of this study and others. ${ }^{(5,6)}$ haloperidol does not appear to be useful in the prevention of delirium in the hospitalized older adult.
A quality improvement initiative focused on early, daily, and progressive mobilization has more patients out of bed and shortens length of stay.

Liu B, Moore JE, Almaawiy U, et al. Outcomes of Mobilisation of Vulnerable Elders in Ontario (MOVEON): a multisite interrupted time series evaluation of an implementation intervention to increase patient mobilisation. Age Ageing. 2018;47(1):112-19.

\section{Background}

Despite evidence suggesting early mobilization reduces length of stay and improves patients' functional status, hospitalized older adults spend the majority of their time in bed. ${ }^{(7)}$

\section{Methods}

This pragmatic, quasi-experimental interrupted time series study of 12,490 patients (mean age $=80$ ) across 14 hospitals evaluated the impact of a complex intervention on patient mobilization. Local teams, consisting of a physician leader, education coordinator, and research coordinator, were recruited to broadcast and implement three key messages: patients should be assessed for mobilization status within 24 hours of admission; mobilization should occur at least three times daily; and mobility should be progressive and scaled and tailored to the patient's ability. The teams were supported by coaching, educational tools, and implementation tools such as checklists and mobility algorithms. Research funding was reserved for evaluation and coaching, with hospitals providing in-kind support for their local team. Mobilization was defined as being out of bed that day, and was determined by the research coordinator during twice weekly audits that took place three times daily.

\section{Findings}

In the post-intervention period, $10.56 \%$ more patients were out of bed $(95 \%$ CI $4.94-16.18, p<.001)$ compared to the pre-intervention period. Length of stay was shortened by 6.1 days $(95 \%$ CI $1.2-11, p=.015)$ in the post-intervention period compared to the pre-intervention period.

\section{Cautions}

This study did not include an internal control to help determine if other factors influenced mobility and length of stay. Limited data were available on falls and injurious falls, though three hospitals had data demonstrating no difference in patients experiencing falls (risk difference $1.2 \%, p=.79$ ). Other downstream outcomes such as functional status, delirium, and stasis ulcers were not collected.

\section{Implications}

Hospitals looking to increase patient mobilization and reduce length of stay can access the free resources available at MOVE Canada (http://movescanada.ca). 


\section{The cardiovascular and mortality benefits of intensive blood pressure treatment persist in the subgroup of adults over the age of 75 in the SPRINT trial (SPRINT- SENIOR).}

Williamson JD, Supiano MA, Applegate WB, et al. Intensive vs. standard blood pressure control and cardiovascular disease outcomes in adults aged $\geq 75$ years. JAMA. 2016;315(24):2673-82.

\section{Background}

Hypertension Canada revised their blood pressure targets in 2017 to reflect the findings of the 2015 Systolic Pressure Intervention Trial (SPRINT trial), which demonstrated reduced cardiovascular events ( 5.2 vs. $6.8 \%$, HR $0.75,95 \%$ CI $0.64-0.89, p<.001, \mathrm{NNT}=61)$ and mortality $(3.3$ vs. $4.5 \%$, HR $0.73,95 \%$ CI $0.60-0.90, p=.003, \mathrm{NNT}=90$ ) over 3.26 years by targeting a systolic blood pressure of less than 120 $\mathrm{mmHg}$ versus less than $140 \mathrm{mmHg}$ in high-risk individuals. Approximately $28 \%$ of the patients in the SPRINT trial were over the age of 75 , but a detailed subgroup analysis of the older adults recruited to the study was not included in the original publication.

\section{Methods}

This pre-specified subgroup analysis examined patients over the age of $75(n=2,636)$ in the SPRINT trial to determine whether a systolic blood pressure target of less than 120 $\mathrm{mmHg}$ reduced major adverse cardiac events. Notable exclusion criteria included diabetes, eGFR $<20$, prior stroke, symptomatic heart failure in the last six months, left ventricular ejection fraction $<45 \%$, dementia, expected survival $<3$ years, $>10 \%$ unintentional weight loss in the past six months, SBP $<110 \mathrm{mmHg}$ following 1 minute of standing, or residing in a nursing home. They also monitored for severe adverse events: hypotension, syncope, injurious falls, electrolyte abnormalities, bradycardia, and hospitalization with acute kidney injury. They conducted secondary exploratory analyses stratified by gait speed and frailty.

\section{Findings}

They had a cohort of slightly frail, but cognitively intact, community-dwelling older adults, with a mean age of 79.9 years, $38 \%$ female, mean frailty index score 0.18 (nonvalidated scale, fit $\leq 0.1$, less fit $>0.1$ to $\leq 0.21$, frail $>0.21$ ) ${ }^{(8)}$, MoCA average of 22 , and $28 \%$ with a gait speed of $<0.8$ $\mathrm{m} / \mathrm{s}$. Over the 3.14 year follow-up period, there were fewer cardiovascular events $(7.74 \%$ vs. $11.2 \%$, HR $0.66,95 \% \mathrm{CI}$ $0.51-0.85, p=.001, \mathrm{NNT}=27)$ and fewer deaths $(5.54 \%$ vs. $8.11 \%$, HR $0.67,95 \%$ CI $.49-0.91, p=.009, \mathrm{NNT}=41)$ in the intensive treatment group compared to the control group. The mean blood pressure achieved in the treatment group was
$123.4 \mathrm{mmHg}$ compared to $134.8 \mathrm{mmHg}$ in the control group. Participants in the intervention arm received on average 1 additional medication. Event rates were higher overall in frailer patients and those with slower gait speed, but the intervention resulted in fewer events regardless of stratification. All serious adverse events except falls occurred more often in the intervention arm, but none reached statistical significance.

\section{Cautions}

The trial did not include nursing home residents, did not stratify age beyond greater than 75 , and the overall cohort was not frail. This study was a pre-specified subgroup analysis of a larger trial, and such subgroup analyses are often considered hypothesis-generating rather than practice-changing.

\section{Implications}

The treatment of blood pressure to a target of $120 \mathrm{mmHg}$ can be considered in fit, ambulatory older adults without significant comorbidities with a goal to reduce major cardiovascular events. Such an approach requires a cautious approach to patient selection that includes consideration of frailty, co-morbidity, potential adverse events from multiple antihypertensive medications and, most importantly, patient preference to adhere to the treatment strategy aligned with their specific goals of care.

A separate meta-analysis of trials including only patients over the age of 65 finds cardiovascular benefits to intensive blood pressure reduction and a nonsignificant increase in the incidence of renal injury.

Bavishi C, Bangalore S, Messerli FH. Outcomes of intensive blood pressure lowering in older hypertensive patients. J Am Coll Cardiol. 2017;69(5):486-93.

\section{Methods}

This meta-analysis restricted its search to include only studies of intensive (defined as systolic blood pressure less than 140 $\mathrm{mmHg}$ ) versus standard blood pressure control in patients over the age of 65 , with cardiovascular events, mortality, and adverse events as outcomes. They identified 22 potential trials, but only 4 trials $(n=10,857)$ were eventually included, as many did not provide separate results for older adults. Three trials compared a target systolic pressure $<140 \mathrm{mmHg}$ versus $>140 \mathrm{mmHg}$, and the fourth trial was the SPRINT-SENIOR trial comparing a target of $<120 \mathrm{mmHg}$ to $<140 \mathrm{mmHg}$.

\section{Findings}

An intensive blood pressure strategy decreased major adverse cardiovascular events (3.7 vs. 5.2\%, RR $0.71,95 \%$ CI 0.60 $-0.84, p=.0001)$, cardiovascular mortality (1.1 vs. $1.7 \%$, 
RR $0.67,95 \%$ CI $0.45-0.98, p=.04)$, and the incidence of heart failure (1.3 vs. $2.0 \%$, RR $0.63,95 \%$ CI $0.40-0.99, p=$ $.04)$. There was an increase in the incidence of renal failure $(1.1 \%$ vs. $0.6 \%$, RR $1.81,95 \%$ CI $0.86-3.60, p=.12)$ and serious adverse events $(25.1 \%$ vs. $24.7 \%$, RR $1.02,95 \%$ CI $0.94-1.09, p=.69)$ in the intensively treated group, but this difference was not statistically significant.

\section{Cautions}

Though efficacy was demonstrated in this meta-analysis for a systolic target less than $140 \mathrm{mmHg}$ in older adults, the authors made note of the limited adverse event data and statistically insignificant increase in renal failure. Due to the age cut-off of greater than 65 , this trial did not include the ACCORD-BP which had a mean age of 62.2 years old. ${ }^{(9)}$ The ACCORD-BP trial recruited 4,733 patients with type 2 diabetes, targeted a blood pressure of $<120 \mathrm{mmHg}$ versus $<140 \mathrm{mmHg}$ and found no reduction in major cardiovascular events.

\section{Implications}

The results of this meta-analysis were in the same direction as the SPRINT-SENIOR trial, which contributed a large proportion of the sample included in the meta-analysis. Avoidance of harm and possible adverse events such as renal failure is important when considering intensive blood pressure control in older adults without frailty.

Secondary subgroup analysis of data from the ALLHAT-LLT trial finds no difference in cardiovascular events in hyperlipidemic adults over the age of 65 treated with pravastatin, and an increased, but nonsignificant, risk of harm.

Han BH, Sutin D, Williamson JD, et al. Effect of statin treatment vs. usual care on primary cardiovascular prevention among older adults: The ALLHAT-LLT Randomized Clinical Trial. JAMA Intern Med. 2017;177(7):955-65.

\section{Background}

While there is summary level data of benefit of statins in secondary prevention of cardiovascular events among older adults ${ }^{(10)}$ the evidence to support their use for primary prevention is less clear.

\section{Methods}

This subgroup analysis drew from the ALLHAT-LLT trial, a randomized controlled component trial of the larger ALLHAT study examining the effect of choice of initial antihypertensive in adults over the age of 55 with hypertension. A subgroup of 2,867 adults over the age of 65 (mean age 71.3 years, $50 \%$ female) who were free of atherosclerotic cardiovascular disease and statin naïve were followed for a mean 4.7 years. All had baseline low density lipoprotein (LDL) between 3.1 and 4.9 (mean $3.8 \mathrm{mmol} / \mathrm{L}$ ) and triglycerides under $4.0 \mathrm{mmol} / \mathrm{L}$ and were started on pravastatin $40 \mathrm{mg}$ by mouth daily or placebo.

\section{Findings}

In patients aged 65 to 74, there was no significant difference in coronary events amongst those treated with pravastatin (HR $0.85,95 \%$ CI $0.63-1.16, p=.31)$ and no significant difference in all-cause mortality (HR 1.08, 95\% CI $0.97-1.42, p=.9$ ). Similar results were found in patients over the age of 75 , with no significant difference in coronary events (HR $0.70,95 \%$ CI $0.43-1.13, p=.14$ ) or all-cause mortality (HR $1.36,95 \% \mathrm{CI}$ $0.98-1.89, p=.24)$. Patients in the intervention arm at six years achieved a lower mean LDL than the usual care group ( 2.82 vs. $3.34 \mathrm{mmol} / \mathrm{L} ; p$ value not reported).

\section{Cautions}

In the over 75 year old group, more patients in the usual care group were taking an antihypertensive medication (86.7 vs. $92.9 \%, p=.01)$ and had a lower mean blood pressure (150.6 vs. $147.5 \mathrm{mmHg}, p=.01)$. Also, significant cross-over occurred: $22.2 \%$ of patients in the intervention group discontinued a statin by the six-year mark, and $29.0 \%$ of patients in the usual group started a statin. Both of these findings bias the result towards an absence of efficacy.

\section{Implications}

This subgroup analysis provides support for the hypothesis that statins in hyperlipidemic patients over the age of 65 provide no benefit. The STAtin therapy for Reducing Events in the Elderly (STAREE) trial should provide better evidence to guide clinical practice for primary prevention. (10) They are randomizing community-dwelling participants over the age of 70 without cardiovascular disease, diabetes, or dementia to atorvastatin $40 \mathrm{mg}$ daily or placebo, with a primary outcome of death, dementia, disability or major cardiovascular event. Study completion is projected to be December 2022.

Comprehensive geriatric assessment (CGA)-based allocation of older patients with advanced non-small cell lung cancer resulted in more fit patients receiving intensive therapy and more frail patients receiving best supportive care without a change in survival and reduced treatment toxicity.

Corre R, Greillier L, Le Caër H, et al. Use of a comprehensive geriatric assessment for the management of elderly patients with advanced non-small-cell lung cancer: the Phase III Randomized ESOGIA-GFPC-GECP 08-02 Study. J Clin Oncol. 2016;34(13):1476-83. 


\section{Background}

Guidelines in the treatment of metastatic non-small cell lung cancer recommend platinum-based doublet therapy (e.g., carboplatin with pemetrexed or gemcitabine) in fit older patients, and single agent therapy (e.g., docetaxel) in the less fit, generally determined by the Eastern Cooperative Oncology Group (ECOG) performance status. It is unknown whether allocation to chemotherapeutic regimens based on comprehensive geriatric assessment would result in better outcomes as compared to performance status alone.

\section{Methods}

This was a multi-center randomized controlled trial of allocation to chemotherapy for stage IV non-small cell lung carcinoma based on performance status and age versus comprehensive geriatric assessment (CGA). The primary outcome was treatment failure-free survival (TFFS), disease progression, or death. They recruited 494 patients (median age 77). Standard allocation assigned patients under the age of 75 and with ECOG of 1 or less to carboplatin-based doublet therapy and others to single agent docetaxel. The CGA-guided allocation arm relied on a comprehensive geriatric assessment to identify the presence of activity of daily living (ADL) or instrumental activity of daily living (IADL) impairment, cognitive impairment via the Mini Mental Status Examination (MMSE), depression defined by Geriatric Depression Scale 5 (GDS5), comorbidity or other geriatric syndromes (falls, dementia, incontinence). Well patients (ECOG 0 to 1, without ADL or IADL impairment, cognitive impairment, significant comorbidity or depression) were assigned to doublet therapy. The presence of co-morbidity (Charlson Co-morbidity Index 2-3), depression (GDS5 2-3) or IADL impairment identified patients as vulnerable, with subsequent allocation to single-agent treatment, while the presence of ADL impairment, impairment in 2 or more IADLs, geriatric syndromes, significant depression (GDS $\geq 4$ ) or an MMSE $<23$ categorized patients as frail and subsequent allocation to best supportive care.

\section{Findings}

There was no significant difference between the CGA and standard allocation groups in TFFS time (3.1 vs. 3.2 months, HR $0.91,95 \%$ CI $0.76-1.1$ ). More patients received doublet therapy in the CGA arm (45.7 vs. $35.1 \%$ ), and fewer received single-agent therapy ( 31.3 vs. $64.9 \%$ ) due to greater allocation of $23 \%$ of frail patients to best supportive care. Treatment failure due to toxicity was more common ( 4.8 vs. $11.8 \%, p=.007$ ) in the standard arm. There were fewer adverse events in the CGA allocation arm (85.6 vs. $93.4 \%$, $p=.015)$.

\section{Cautions}

The CGA was oncologist-led and did not include management of identified problems, which is not in the spirit of a comprehensive geriatric assessment completed by geriatricians or Care of the Elderly physicians, which would typically include both assessment and intervention. The study intervention was more in keeping with the use of a more refined set of criteria for frailty (and hence better patient selection) than age and ECOG status alone. Despite these limitations, interestingly, no decrement in survival was seen despite one-third of the CGA arm receiving best supportive care.

\section{Implications}

Comprehensive geriatric assessments in oncology patients have not been shown to improve survival. The reduced toxicity seen in the CGA arm implies less overtreatment (perhaps due to allocation of more frail adults to supportive care), but higher survival could potentially be achieved through a more aggressive allocation algorithm that assigns more patients to singlet therapy. Ongoing study into how best to incorporate comprehensive geriatric assessment to optimize the care of cancer patients continues to unfold. As of January 2018, there are 144 trials registered with ClinicalTrials.gov evaluating comprehensive geriatric assessment in cancer patients.

A randomized controlled trial of pre-operative comprehensive geriatric assessment by a multidisciplinary team reduced length of stay and post-operative complications amongst vascular surgery patients when compared to nursing-led, pre-operative assessment.

Partridge JSL, Harari D, Martin FC, et al. Randomized clinical trial of comprehensive geriatric assessment and optimization in vascular surgery. Br J Surg. 2017;104(6):679-87.

\section{Background}

Emerging evidence suggests geriatric pre-operative assessment improves post-operative outcomes.

\section{Methods}

In this first study of comprehensive geriatric assessment prior to vascular surgery (elective aortic aneurysm repair or lower limb arterial bypass), 209 patients over the age of 65 were randomized to either pre-operative CGA and optimization clinic, including geriatrician, clinical nurse specialist, social worker, and occupational therapist, or standard nurse-led, pre-operative assessment. Primary outcome was hospital length of stay, with secondary outcomes including incidence of delirium and other post-operative complications, as well as new diagnoses and management decisions made at preoperative assessment. 


\section{Findings}

Hospital length of stay was reduced in the CGA group (3.32 vs. 5.53 days, $95 \%$ CI $0.46-0.79, p<.001)$. There were fewer episodes of delirium ( 11 vs. $24 \%, p=.018$ ), cardiac complications such as acute coronary syndrome, heart failure tachyarrhythmia, or bradyarrhythmia ( 8 vs. $27 \%, p=.001$ ) and bowel/bladder complications such as urinary tract infection, constipation or incontinence (33 vs. 55\%, $p=.003$ ). Considerably more patients in the CGA arm received new diagnoses of cognitive impairment $(46.5$ vs. $1 \%, p<.001)$, chronic kidney disease $(25.7$ vs. $0 \%, p<.001)$, and COPD $(14.9$ vs. $0 \%, p<.001)$ with substantially more changes to medications being made ( 86.1 vs. $4.0 \%, p<.001$ ), compared to the standard pre-operative assessment arm.

\section{Cautions}

The usual care arm in this British study was a nurse-led, pre-operative assessment which is generally not the structure of Canadian pre-operative clinics with high-risk vascular patients. Therefore, generalizing these results to a Canadian setting is difficult. Additionally, a significant risk of bias was evident since outcomes were assessed by unblinded assessors.

\section{Implications}

The evidence base for reduced length of stay through preoperative comprehensive geriatric assessment continues to grow and now includes the vascular surgical setting.

\section{CONFLICT OF INTEREST DISCLOSURES}

The authors declare that no conflicts of interest exist.

\section{REFERENCES}

1. McKeith IG, Dickson DW, Lowe J, et al. Diagnosis and management of dementia with Lewy bodies: third report of the DLB Consortium. Neurology. 2005;65(12):1863-72.
2. McKhann GM, Knopman DS, Chertkow H, et al. The diagnosis of dementia due to Alzheimer's disease: Recommendations from the National Institute on Aging-Alzheimer's Association workgroups on diagnostic guidelines for Alzheimer's disease. Alzheimers Dement. 2011;7(3):263-69.

3. Petersen RC. Mild cognitive impairment as a diagnostic entity. J Intern Med. 2004;256(3):183-94.

4. Schrijver EJ, de Vries OJ, Verburg A, et al. Efficacy and safety of haloperidol prophylaxis for delirium prevention in older medical and surgical at-risk patients acutely admitted to hospital through the emergency department: study protocol of a multicenter, randomised, double-blind, placebo-controlled clinical trial. BMC Geriatr. 2014;14:96.

5. Kalisvaart KJ, De Jonghe JFM, Bogaards MJ, et al. Haloperidol prophylaxis for elderly hip-surgery patients at risk for delirium: a randomized placebo-controlled study. J Am Geriatr Soc. 2005;53(10):1658-66.

6. Siddiqi N, Harrison JK, Clegg A, et al. Interventions for preventing delirium in hospitalised non-ICU patients. Cochrane Database Syst Rev. 2016;3:CD005563.

7. Brown CJ, Redden DT, Flood KL, et al. The underrecognized epidemic of low mobility during hospitalization of older adults. $J$ Am Geriatr Soc. 2009;57(9):1660-65.

8. Pajewski NM, Williamson JD, Applegate WB, et al. Characterizing frailty status in the systolic blood pressure intervention trial. J Gerontol A Biol Sci Med Sci. 2016;71(5):649-55.

9. The ACCORD Study Group. Effects of intensive blood-pressure control in type 2 diabetes mellitus. $N$ Engl J Med. $2010 \mathrm{Apr}$ 29;362(17):1575-85.

10. Afilalo J, Duque G, Steele R, et al. Statins for secondary prevention in elderly patients: a hierarchical Bayesian meta-analysis. $J$ Am Coll Cardiol. 2008;51(1):37-45.

Correspondence to: Dov Gandell, MDCM, FRCPC, Sunnybrook Health Sciences Centre, HG-69 - 2075 Bayview Ave., Toronto, ON M4N 3M5, Canada

E-mail: dov.gandell@sunnybrook.ca 